\title{
Cerebral dural arterio-venous fistula - Part II: Endovascular therapy
}

\author{
Michael Hofko ${ }^{1}$, Michael Sonnberger ${ }^{2}$ and Franz A Fellner ${ }^{1,3}$ \\ ${ }^{1}$ Central Radiology Institute, Kepler University Hospital, Medical Faculty of the Johannes Kepler University, Linz, Austria \\ ${ }^{2}$ Institute of Neuroradiology, Kepler University Hospital, Medical Faculty of the Johannes Kepler University, Linz, Austria \\ ${ }^{3}$ Medical Faculty of the Friedrich-Alexander-University of Erlangen-Nürnberg, Erlangen, Germany
}

\begin{abstract}
Case report of an acute symptomatic 60y-year-old male patient with an occipital dural arteriovenous fistula, classified as Cognard Typ IV, and consecutive treatment by transfemoral cerebral angiography and embolization with liquid embolization material (Onyx).
\end{abstract}

\section{Introduction}

Cerebral dural arteriovenous fistula (DAVF) is a type of AVM in which there is a communication between dural arteries and cerebral venous sinuses. These lesions constitute $10-15 \%$ of all cerebral AVMs and most of them seem to be acquired, only few are congenital. There is a female to male ratio of 2:1 and most of them are diagnosed in the fifth and sixth decade. The distinguishing feature between DAVF and cerebral AVM is the fact that there is no parenchymal nidus and there is a dural arterial supply [1].

We report a 60-year-old man with acute diagnosis of an occipital dural arteriovenous fistula classified Cognard Typ IV, treated by transfemoral cerebral angiography and embolization with liquid embolization material (Onyx - composed of a mixture of ethylenevinyl alcohol copolymer suspended in the solvent dimethyl sulfoxide (DMSO) - Tantalum is added for radiopacity).

\section{Case report}

We present a case of a 60 -year old male patient with recent diagnosis of an occipital dural arteriovenous fistula classified Cognard Typ IV, treated by transfemoral cerebral angiography and embolization with liquid embolization material (Onyx).

Endovascular therapy was performed in intubation anesthesia, the interventional neuroradiologist (M.S.) used the right-sided common femoral artery as an arterial access. Via a 6-F-vascular lock a 6-F-catheter was positioned in the left maxillary artery (Figure 1).

Furthermore, a distal access catheter was placed at the branch point of the middle meningeal artery (Figure 2). Using this guiding catheter, a microcatheter was positioned at the origin of the fistula.

After the microcatheter has been brought to the optimal end position, the fistula was completely occluded with two ampoules of Onyx (ethylene vinyl alcohol copolymer) (Figure 3).

The final digital subtraction images showed complete occlusion of the dural arteriovenous fistula (Figure 4).

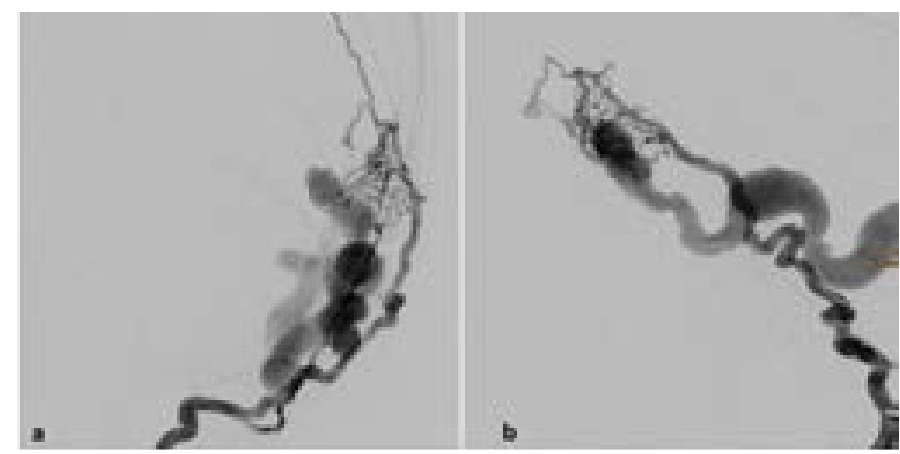

Figure 1. Dural arterio-venous fistula: Catheter is placed in the left maxillary artery. a) ap view; b) lateral view

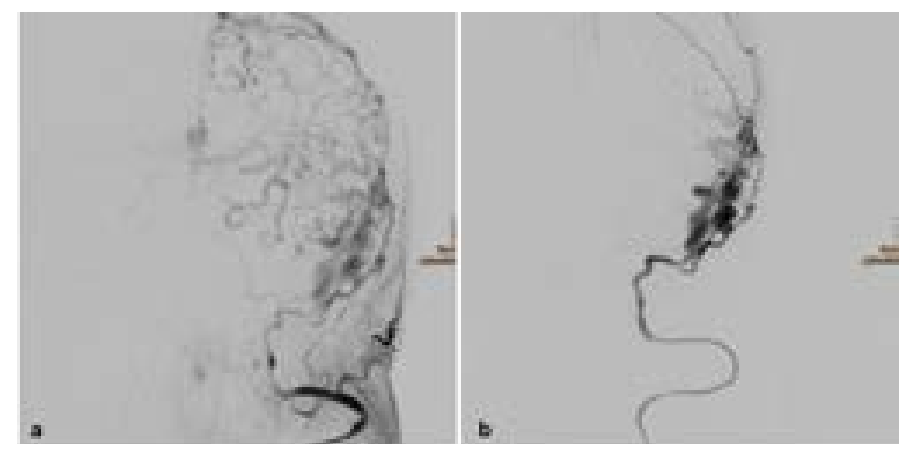

Figure 2. $\mathrm{a} / \mathrm{b}$ showing the distal access catheter at the branch point of the middle meningeal artery

*Correspondence to: Michael Hofko, Central Radiology Institute, Kepler University Hospital, Medical Faculty of the Johannes Kepler University, Linz, Austria, E-mail: michael.hofko@kepleruniklinikum.at

Key words: cerebral dural arterio-venous fistula, digital subtraction angiography, endovascular therapy, interventional radiology, embolization, onyx, computed tomography (CT)

Received: April 16, 2020; Accepted: May 18, 2020; Published: May 21, 2020 


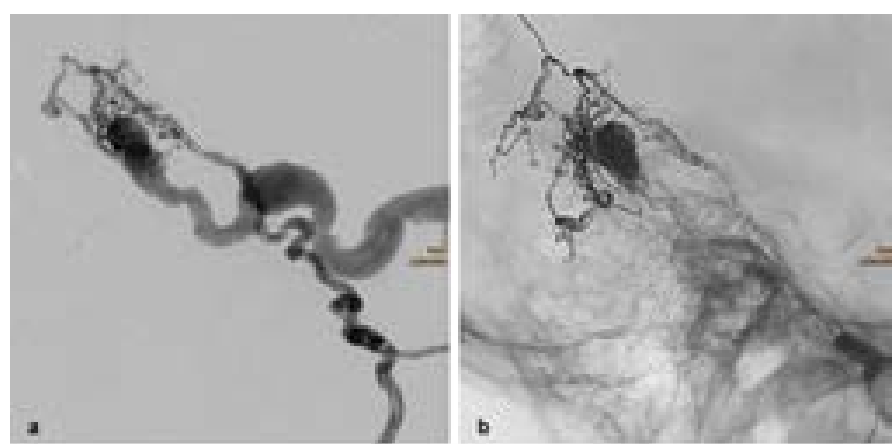

Figure 3. Interventional procedure. a) Positioning of the microcatheter in front of the arterial feeders; b) Digital subtraction angiography showing the onyx cast with complete obliteration of the fistula

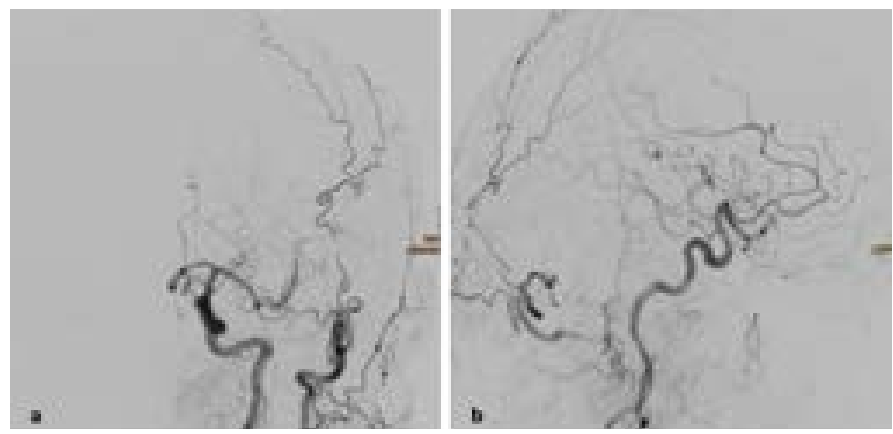

Figure 4. Final series of the interventional procedure. The digital subtraction angiography images of the left common carotid artery show that there is no residual fistula. a) ap-view, b) lateral view

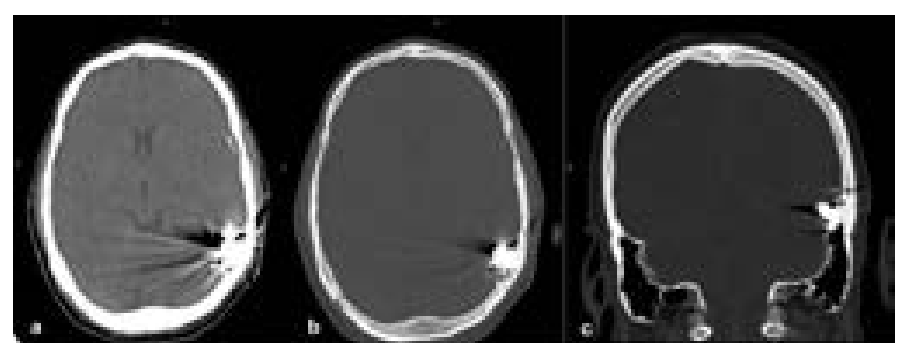

Figure 5. Non-enhanced cranial CT scan after the interventional procedure: The hyperdense embolization material produces strong artefacts. No hemorrhage. No territorial infarction. a) soft tissue window, transverse, b bone window, transverse, c bone window, coronal reformation

Immediately after the intervention a cerebral non contrast enhanced computed tomography was performed, there were strong artefacts due to the embolization material, but no bleeding or territorial infarction after the intervention (Figure 5).

\section{Discussion}

The applied technique of transarterial embolization is ideally used for high-grade DAVFs, such as those with direct cortical venous drainage, or in situations in which venous access is limited.

Superselective microcatheter angiography, three-dimensional rotational angiography and high-resolution flat-panel computed
Table 1. Classification of dural arteriovenous fistulas (Cognard) [2]

I Normal antegrade flow into dural sinus

a. Retrograde flow into sinus(es)

II b. Retrograde filling of cortical vein(s)

c. Retrograde drainage into sinus(es) and cortical veins

III Direct drainage into cortical veins without venous ectasia

IV Direct drainage into cortical veins with venous ectasia $>5 \mathrm{~mm}$ and $3 \mathrm{x}$ larger than diameter of draining vein

V Drainage to spinal perimedullary veins

tomography, such as "DynaCT", are recommended to evaluate the arterial and venous anatomy of a DAVF both before and after embolization. To prevent premature polymerization and contamination of diagnostic catheters and solutions, embolic agents such as Onyx should be handled on a separate table when not in use. Furthermore, a separate set of gloves should be used prior to handling these agents and at the end of the procedure prior to final diagnostic angiography [1].

Onyx is one possible material for liquid embolization, it is a mixture of ethylene-vinyl alcohol copolymer suspended in the solvent dimethyl sulfoxide (DMSO) - Tantalum is added for radiopacity. First reports were published in 1990, in Europe this drug is available since 1999.

Transarterial embolization is recommended in dural arteriovenous fistulas Cognard Typ IIb - V (Table 1).

There are some technical advantages when using Onyx:

1. There is less operator dependence concerning the interventional radiologist.

2. No wedged microcatheter position is required.

3. Different feeders can be occluded with a single injection from a single pedicle.

For planning the intervention the following points should be considered.

1. Where is the location of the fistula point/points?

2. What is the location of the feeding arteries?

3. How is the venous drainage pattern?

4. Where are the most promising pedicles to be injected?

After the embolization procedure an immediate cerebral CT scan is recommend. Furthermore, due to dural involvement, pain medication is very important and low-molecular weight heparin should be prescribed for 1 to 4 weeks to avoid venous thrombosis [1].

\section{Disclosure}

No conflicts of interest.

\section{References}

1. Colby GP, Paul AR, Ciceri EF, Coon AL (2012) Endovascular Management of Dural Arteriovenous Fistulas. In: Operative Neurosurgical Techniques: Indications, Methods, and Results. 6th edition 1: 1079-1088.

2. Jabbour P, Tjoumakaris S, Chalouhi N, Randazzo C, Gonzalez LF, et al. (2013) Neuroimag Clin N Am 23: 625-636.

Copyright: (C2020 Hofko M. This is an open-access article distributed under the terms of the Creative Commons Attribution License, which permits unrestricted use, distribution, and reproduction in any medium, provided the original author and source are credited. 